\title{
Some Methods of Evaluating Eigenvalues of Symmetric Matrix Ding $\mathrm{YaO}^{1, \mathrm{a}}$
}

${ }^{1}$ The Marx doctrine and the General Education College, Chongqing College of Electronic Engineering, Shapingba, Chongqing China 401331

adingyao628@126.com

Keywords: Real symmetric matrix, Real symmetric triangle matrix, Eigenvalue, Jacobi method; DC method, QR method

\begin{abstract}
This article introduces some methods to evaluate eigenvalues of the real symmetric matrix, especially for real symmetric triangle matrix. For these methods, we give concrete steps. Finally, by comparing these methods, we study advantages of various methods.
\end{abstract}

\section{Introduction}

The eigenvalue problem of matrix is often used for scientific computing and engineering problems, and has important application in the field of mathematical physics, geophysics, optical, mechanical, structural design and optimization. It apparently is a question of simple nonlinear equations $A x=\lambda x$, of which $\lambda$ is the characteristic value of $A$. In order to solve $\lambda$, a simple idea is to solve the equation $\operatorname{det}(A-\lambda I)=0$.Unless for individual special matrix, this method is not feasible for general matrix. First of all, if the order of $A$ is large, the calculation amount of determinant $\operatorname{det}(A-\lambda I)$ will be very large. Secondly, there is no a general method which can be used to find out the roots of polynomials. Based on the above reasons, people try to find other more convenient way.

In this paper we introduce the Jacobi method, QR method, the classic divide and conquer. These algorithms are promoted in the use of real symmetric matrix, as well as analysis and comparison between algorithms.

\section{The basic method and main conclusions}

Jacobi iterative method. The Jacobi method was proposed in 1846[1]. The Jacobi method is used to calculate all the eigenvalues of a real symmetric matrix and corresponding feature value vector by a transformation method. The basic idea is to put a symmetric matrix $A$ into a diagonal matrix through a set of plane rotation (orthogonal similarity transformation), and obtain all eigenvalues.

The steps of Jacobi process:

Make $A=A_{0}$, sequentially constructed matrix sequence $A_{k}$, make $A_{k}=R^{T}{ }_{k}(p, q) A_{k-1} R_{k}(p, q)$ $(k=1,2 \cdots)$, of which $R(p, q)$ is turned in the $(p, q)$ plane angle of a rotating $\theta$. Among them: $\mathrm{R}_{\mathrm{k}}(\mathrm{i}, \mathrm{i})=1, \mathrm{i} \neq \mathrm{p}, \mathrm{q} \mathrm{R}_{\mathrm{k}}(\mathrm{i}, \mathrm{i})=\cos \theta, \mathrm{i}=\mathrm{p}, \mathrm{q} \quad \mathrm{R}_{\mathrm{k}}(\mathrm{i}, \mathrm{j})=-\mathrm{R}_{\mathrm{k}}(\mathrm{j}, \mathrm{i})=\sin \theta, \mathrm{i}=\mathrm{p}, \mathrm{j}=\mathrm{q}$ $R(i, j)=0, i, j=$ other.

Strategies which are used in the selecting plane and determining the angle $\theta$ of rotation are simple. The term of maximum model $a_{p q}{ }^{(s)}$ is determined by seeking elements of $A_{s}$ located above the main diagonal (taking into account the symmetry, we only need to seek from the elements from the upper part in matrix A). Then, select rotation angle $\theta$, make $a_{p q}{ }^{s+1}=0$. Due to the similar transformation of plane rotating $R(p, q)$ simply affect located in the $p, q$ row and column element. 
By Jacobi algorithm, finally a diagonal matrix with a certain precision can be obtained, so it is $R A R^{T}=D$, where each column of $R^{T}$ is feature vector of the matrix $A$. Jacobi method is an iterative method used for calculating all the characteristics value and eigenvector of symmetric matrix $A$, higher accuracy, but the amount of calculation is great[2].

QR algorithm. The two methods, QR algorithm and divide-and-conquer methods, are used for characteristic value calculation of symmetric tridiagonal matrix. Therefore, when we introduce QR and divide-and-conquer methods, if there is no special instruction, the matrix has been turned into symmetric tridiagonal matrix. Then we introduce the QR algorithm ${ }^{[3]}$ :

By the algebra, if the matrix $A$ is a non-singular matrix, then $A$ can be decomposed into the product of an orthogonal matrix $Q$ and upper triangular matrix $R$, namely, QR decomposition of $\quad$ A: $\quad A=Q R \quad$ Thus a new matrix $\quad B=R Q=Q^{T} A Q \quad A_{s+1}=Q_{s}^{*} A_{s} Q_{s} \quad=Q_{s}^{*} Q_{s-1}^{*} A_{s-1} Q_{s-1} Q_{s}$ $=\left(Q_{s}^{*} \cdots Q_{1}^{*}\right) A_{1}\left(Q^{(s)} R^{(s)}=A_{1}^{s} Q_{1} \cdots Q_{s-1} R_{s-1} \cdots R_{j}=A_{1} Q^{(s-1)} R^{(s-1)} R Q_{s} A_{s+1}=A_{1} Q_{1} Q_{2} \cdots Q_{s}\right)$ is obtained.

Obviously, $\mathrm{B}$ is obtained through orthogonal similarity transformation by $\mathrm{A}$, so $B$ and $A$ have the same characteristic value. B is QR decomposition again, and a new matrix is obtained. This process is repeated to obtain a matrix sequence. QR algorithm is defined by the sequence $A_{s}=Q_{s} R_{s}, A_{s+1}=R_{s} Q_{s}$. Of course, in the practical application, Emirates matrix $\mathrm{Q}$ is determined, and make $Q_{s}^{*} A_{s}=R_{s}$. Because of $A_{s+1}=R_{s} Q_{s}=Q_{s}^{*} A_{s} Q_{s}$, so matrix sequence $A_{s}$ is similar to each other. If $A_{1} \equiv A$, we can get: $Q^{(s)} R^{(s)}=Q_{1} \cdots Q_{s-1} Q_{s} R_{s} R_{s-1} \cdots R_{1}=Q_{1} \cdots Q_{s-1} A_{s} R_{s-1} \cdots R_{1}$ $=A_{1} Q_{1} \cdots Q_{s-1} R_{s-1} \cdots R_{1}=A_{1} Q^{(s-1)} R^{(s-1)}$. Finally, we can get $Q^{(s)} R^{(s)}=A_{1}^{s}$.

Thus, $Q^{(s)}$ and $R^{(s)}$ give the decomposition of $A_{1}^{s}$. If the diagonal elements of upper triangular matrix $R_{j}$ are required to positive, then the decomposition is unique. QR algorithm has a very important property: if the $A$ is three diagonal matrix, then all the matrix $\mathrm{A}$ is tridiagonal, and if $A$ is non-singular matrix, then $\left\{A_{k}\right\}$ generated by $Q R$ algorithm converges to a diagonal matrix ${ }^{[4]}$.

Divide and conquer. Divide and conquer (DC) algorithm, was first proposed by Dongarra and Sorensen in 1987,. The basic steps are:

(1) Divide matrix A: set A is a real symmetric three diagonal matrix,

$A=\left[\begin{array}{ccccc}a_{1} & b_{1} & & & \\ b_{1} & a_{2} & b_{2} & & \\ & \ddots & \ddots & \ddots & \\ & & b_{n-2} & a_{n-1} & b_{n-1} \\ & & & b_{n-1} & a_{n}\end{array}\right]$, division of $\mathrm{A}: A=A^{\prime}+\mathrm{b}_{\mathrm{k}} \mathrm{zz}^{\mathrm{T}}, A^{\prime}=\left[\begin{array}{cc}A_{1} & \\ & A_{2}\end{array}\right]$. Among them , $\mathrm{Z}=(0, \cdots, 0,1,1,0, \cdots, 0)^{\mathrm{T}}$ (1 are respectively located in the $\mathrm{K}$ line and the $\mathrm{k}+1$ line) ,thus, the $\mathrm{A}$ is divided into a partitioned matrix and a matrix with a rank of 1 .

(2) Glue. $A_{1}$ and $A_{2}$ are sub-matrix blocks obtained after dividing. Suppose eigenvalues of $A$ and $B$ have been determined. Namely, assume get orthogonal matrix $Q_{1}, Q_{2}$, make $A_{1}, A_{2}$ respectively translated into diagonal matrix $\mathrm{D}_{1}, \mathrm{D}_{2}: \mathrm{Q}_{1} \mathrm{~A}_{1} \mathrm{Q}^{\mathrm{T}}{ }_{1}=\mathrm{D}_{1}, \mathrm{Q}_{2} \mathrm{~A}_{2} \mathrm{Q}^{\mathrm{T}}{ }_{2}=\mathrm{D}_{2}$, referred to as $\mathrm{Q}=\left[\begin{array}{ll}\mathrm{Q}_{1} & \\ & \mathrm{Q}_{2}\end{array}\right]$, then $\mathrm{QAQ}^{\mathrm{T}}=\left[\begin{array}{ll}\mathrm{D}_{1} & \\ & \mathrm{D}_{2}\end{array}\right]+\mathrm{b}_{\mathrm{k}} y y^{T}$, among them $\mathrm{y}=\left[\begin{array}{ll}L_{1}, & L_{2}\end{array}\right], L_{1}$ is the last column of $Q_{1}, L_{2}$ is the first column of $Q_{2}$. Note that $Q$ is still orthogonal matrix. So $Q A Q^{T}$ and $A$ have the same characteristic values. And then original problem is transformed into a characteristic value problem of the rank of a diagonal matrix. Then, we are concerned about the problem that how to link the eigenvalues of matrix $A$ and eigenvalues of matrix $A^{\prime}$ [3]. 
The characteristic values of $A_{1}, A_{2}$ offer the inclusion interval of characteristic values of $A$, and each interval contains the only one characteristic value of $A$. By using this feature, we can use the Sturm sequence of three diagonal matrix and the method of parabolic interpolation to calculate the eigenvalues of $A$.

\section{Comparison of algorithms}

After we have understood that various methods of solving matrix eigenvalues and some differences between them, next, we will compare the divide and conquer method and Jacobi method on the run time and number of iterations: look at the following example

Set matrix: $\quad A=\left(\begin{array}{ccccc}4 & -3 & 2 & 6 & 1 \\ -3 & 5 & 7 & 4 & 0 \\ 2 & 7 & 3 & -1 & 8 \\ 6 & 4 & -1 & 7 & -2 \\ 1 & 0 & 8 & -2 & -6\end{array}\right)$

Two methods are now used to find the eigenvalues and eigenvectors of matrix A and B. By running on the computer, the results are obtained as follows:

\begin{tabular}{|c|c|c|}
\hline $\mathrm{A}$ & $\begin{array}{l}\text { Operation time } \\
\text { (seconds) }\end{array}$ & $\begin{array}{c}\text { Iteration number } \\
(\mathrm{n})\end{array}$ \\
\hline Divide and conquer & $\mathrm{t}=2.8$ & $\mathrm{n}=9$ \\
\hline Jacobi & $\mathrm{t}=2.1$ & $\mathrm{n}=35$ \\
\hline
\end{tabular}

From the above table, we can draw the following conclusions:

The method used by divide and conquer is QR method to solve the characteristic value of each sub-block matrix. So it has the advantages of QR method. The algorithm is stable and the calculation precision is higher, at the same time, the method of parabolic interpolation is employed when calculate each characteristic value, and thus it is superlinear converged. And because each sub-blocks matrix characteristic value and each characteristic value caculated aft er gluing are independent, it is possible to calculate them

using different servers and some time would be saved correspondingly. Divide and conquer method is great flexible, that is, it can be used to solve all the eigenvalues and part of the eigenvalues and has great practical value. And its biggest advantage is that it can be implemented in parallel, and the iterative procedure is simple and so divide and conquer method is an ideal algorithm to three diagonal matrixes.

In these three algorithms, due to the Jacobi method and QR method are used to construct matrix sequence, make $P_{k}^{-1} A P_{k}$ converge to a diagonal or three diagonal matrixes. Therefore, these two algorithms cannot be parallel calculated. For real symmetric matrix, the Jacobi method can be used to solve the eigenvalues and eigenvectors of matrix, and divide and conquer method and the QR algorithm can be used to only solve characteristic value. However, for non-symmetric matrix, using divide and conquer algorithm may lead to leakage of root. All characteristic values can be obtained by QR algorithm, and Jacobi method cannot[4].

\section{Conclusion}

In recent years, the parallel computation of matrix eigenvalue problem has been studied. It is the direction of future efforts to find other matrix method. Many effective parallel algorithms are also proposed, such as Homo Topy continuous method, parallel block elimination iterative method, and spectral decomposition algorithm is a typical representative. The method in this paper is only a small part. Each method has its advantages and disadvantages, and there is no universal method for all kinds of problems. It can only be analyzed in detail, so the research prospect in this field is very broad. 


\section{References}

[1] C. G. J . Jacobi. Uber ein leichtes verfahren, die in der Theorie der säkularstörungen vorkommenden Gleichungen numerisch aufzubsen . JKeine Ayew , Math. 1846 ,30 :51 95.

[2] Dai H,Lancaster P. Linear matrix equation from an inverse problem of vibration theory.Linear Algebra and its Applications,1996,(0):31-47.

[3] Paige C C,Saunders M A. Towards a generalized singular value decomposition.SIAM JOURNAL ON NUMERICAL ANALYSIS,1981.405.

[4] Stewart C W. Computing the CS-decomposition of a partitional orthogonal matrix. Numerische Mathematik,1982.298-306. 